\section{Informe sobre la actividad física en horas libres y en horas de trabajo en la población estadounidense ${ }^{1}$}

Los estudios sobre la actividad física de las personas que se han llevado a cabo en el mundo, ya sea en países desarrollados como en países en desarrollo, se han centrado en su mayor parte en el gasto energético vinculado con las actividades realizadas en horas de ocio, o bien en el consumo de energía durante el trabajo y las tareas cotidianas normales, pero raras veces en ambos. Destacan como ejemplos dos estudios incluidos en este número de la Revista: uno sobre los niveles de actividad física de adultos brasileños en sus horas libres (1), y el otro sobre la práctica de los deportes y ejercicios en mujeres mexicanas en edad reproductiva (2). Ambos describen distintos aspectos de las actividades físicas practicadas voluntariamente en horas de ocio: su cantidad, su tipo, los factores que inciden en su práctica y las variables independientes de carácter demográfico y socioeconómico que se asocian con ellas. No obstante, los estudios de este tipo, que aportan información muy reveladora acerca de los hábitos y preferencias de la población en materia de actividad física, solo pueden dar una idea aproximada del gasto energético total que tienen en promedio los habitantes de esos países debido a que no contemplan la actividad física desempeñada como parte del oficio u ocupación o de las demás tareas obligatorias diarias. A la luz de esta limitación, resulta especialmente interesante un informe reciente del Gobierno de Estados Unidos de América, el primero de procedencia gubernamental en estimar el tiempo total dedicado diariamente por los habitantes del país a actividades físicas en horas de ocio y también a las llamadas "tareas cotidianas habituales", es decir, aquellas que obedecen a las exigencias diarias de la vida, como el trabajo y las tareas domésticas.

\section{Los datos y su procedencia}

El informe, que se titula La actividad física en adultos: Estados Unidos, 2000 [Physical Activity Among Adults: United States, 2000], presenta prevalencias estimadas de actividad física a partir de los resultados de la National Health Interview Survey (NHIS) del año 2000. Esta encuesta poblacional de alcance nacional la lleva a cabo cada año el Centro Nacional de Estadísticas de Salud, perteneciente a los Centros para el Control y la Prevención de Enfermedades (CDC), en Atlanta, Georgia, que a su vez forman parte del Departamento de Salud y Servicios Sociales de Estados Unidos (USDHHS). A 
partir de 1975 se empezó a recoger en la NHIS información periódica acerca de las actividades físicas realizadas en horas de ocio por la población adulta estadounidense. Asimismo, en 1985 y 1990 se agregaron preguntas destinadas a cuantificar las actividades físicas pesadas vinculadas con el trabajo o con las tareas cotidianas principales. Finalmente, en 2000 se introdujeron en el Módulo sobre el Control del Cáncer de la NHIS algunas preguntas centradas específicamente en "las tareas cotidianas habituales" (estar sentado, caminar, permanecer de pie, o levantar y cargar objetos), en un intento por cuantificar el nivel de actividad física de los adultos estadounidenses fuera de sus horas de ocio. Las conclusiones del informe se basan en las respuestas a preguntas relacionadas con las tareas cotidianas habituales y también con las actividades físicas en horas de ocio, que juntas constituyen un fiel indicador de la actividad física general que practican a diario, en promedio, las personas mayores de 18 años en los Estados Unidos.

La NHIS, que abarca a una muestra representativa de la población civil estadounidense, se realiza mediante entrevistas a domicilio. En cada entrevista se recoge información demográfica sobre todos los integrantes del hogar, junto con datos más completos obtenidos sobre un solo miembro de familia mayor de 18 años y sobre un niño entre los 0 y 17 años de edad, ambos elegidos aleatoriamente. $\mathrm{Al}$ adulto así escogido se le pide información sobre sí mismo, aunque en casos excepcionales esta información puede ser proporcionada por otro adulto en su lugar. En el año 2000 se encuestó a los miembros de alrededor de 39000 hogares y se recolectaron datos sobre más de 100000 personas en todos los 50 estados del país (3).

\section{Definiciones e indicadores de actividad física en horas de ocio y durante las tareas cotidianas habituales}

Los indicadores de la actividad física total realizada diariamente se basaron en cuatro preguntas acerca de la participación en actividades físicas en horas de ocio y en dos preguntas acerca de las tareas cotidianas habituales, es decir, las realizadas en conexión con el trabajo u otras obligaciones normales de la vida, sin fines recreativos (ir al trabajo, hacer mandados, limpiar la casa, etc.). En el primer caso se indagó acerca de la frecuencia y duración de actividades físicas leves, moderadas o pesadas. Se definió como actividad leve o moderada la que tuviese una duración mínima de 10 minutos y se acompañara de poca sudación o un aumento leve a moderado de la frecuencia cardíaca y respiratoria. La actividad física pesada se definió como aquella que durara como mínimo 10 minutos y que se acompañara de sudación profusa o de una marcada aceleración de la frecuencia cardíaca o respiratoria.

Para definir la actividad física practicada con regularidad en horas de ocio se usaron los siguientes indicadores, basados tanto en la frecuencia como en la duración de la actividad: actividad física leve o moderada cinco veces por semana o más, con una duración mínima de 30 minutos por episodio, o bien una actividad física intensa tres veces por semana o más, durante un mínimo de 20 minutos por episodio. El nivel de actividad física en conexión con las tareas cotidianas habituales se basó en el número de veces que había que "cargar o levantar objetos" en el transcurso del día.

\section{Datos revelados por el informe}

Los resultados del informe se resumen en cuatro cuadros extensos ${ }^{2}$ donde se presentan por separado la actividad representada por las tareas cotidianas habituales y la practicada en ratos de ocio. Al sumar estas actividades se obtiene un indicador del nivel general de la actividad física practicada diariamente.

Según los resultados presentados en el informe, $19 \%$ de todos los adultos en los Estados Unidos tienen un alto nivel de actividad física (que se define como "abundante actividad durante las tareas cotidianas habituales además de actividades físicas practicadas con regularidad en horas de ocio"), mientras que casi la tercera parte $(32,1 \%)$ tiene un nivel moderado. Más hombres (21,3\%) que mujeres $(16,9 \%)$ tienen un alto nivel de actividad física en general, y en ambos sexos dicho nivel se reduce paulatinamente conforme avanza la edad. De hecho, los adultos de uno $\mathrm{u}$ otro sexo pertenecientes al grupo de mayor edad (65 años o más) son cinco veces más propensos que los del grupo más joven (18 a 24 años) a no tener ningún tipo de actividad física. En general, $31,8 \%$ de todos los adultos practican con regularidad alguna actividad física en sus ratos de ocio.

Por otra parte, 1 de cada 4 personas adultas tiene un nivel bajo de actividad física (lo cual significa que es moderadamente activa durante el desempeño de sus tareas cotidianas habituales e inactiva el resto del tiempo) o no tiene ninguna. Mientras más activa es una persona en sus tareas cotidianas

\footnotetext{
2 Los cuatro cuadros aportan la siguiente información para adultos de 18 años o más, según diversas características sociodemográficas y socioeconómicas: cuadro 1: distribución porcentual del tipo de tarea cotidiana habitual en que se camina, se está sentado, o se está de pie; cuadro 2: distribución porcentual del nivel diario de actividad consistente en levantar o cargar bultos; cuadro 3: porcentaje de personas que practican de un modo regular actividades físicas en horas de ocio; cuadro 4: distribución porcentual del nivel de actividad física general.
} 
habituales, bien sea porque camina o levanta y carga objetos pesados o de peso mediano, mayores son las probabilidades de que practique con regularidad alguna actividad física en sus ratos de ocio.

El informe también revela que alrededor de $50 \%$ de todos los adultos caminan durante el desempeño de sus tareas cotidianas habituales, mientras que más de la tercera parte $(36,8 \%)$ permanecen sentados y alrededor de $14 \%$ se mantienen de pie. Casi tres cuartas partes $(74,5 \%)$ de los adultos levantan o cargan bultos moderadamente pesados o pesados durante el desempeño de sus tareas diarias normales.

En el informe también se documenta la actividad física realizada a diario por diferentes grupos poblacionales. Cerca de $15 \%$ de los adultos de ascendencia hispana, independientemente de la raza, $14 \%$ de la población adulta de raza negra y $20 \%$ de los adultos blancos tienen un alto nivel de actividad física.

Según el informe, otros factores también se asocian con el nivel de actividad física, particularmente la escolaridad, el ingreso, el estado civil y la zona geografía donde se vive. En lo que respecta a la escolaridad, alrededor de 1 de cada 4 adultos con un título universitario de posgrado $(4,7 \%)$ tiene un alto nivel de actividad física en general, en comparación con 1 de cada 7 que no completaron la escuela secundaria $(14,4 \%)$. Estos últimos se muestran dos veces más propensos que aquellos a no tener actividad física alguna. Las mujeres con títulos universitarios de posgrado $(26,8 \%)$ son dos veces más proclives a tener un alto nivel de actividad física en general que las mujeres que no acaban la escuela secundaria $(11,2 \%)$.

Las personas adultas con ingresos por debajo del nivel de pobreza $(18,0 \%)$ son tres veces más proclives a no practicar actividad física alguna que los adultos pertenecientes al nivel de mayores ingresos (4 veces o más por encima del nivel de pobreza) $(5,9 \%)$.

Las mujeres casadas $(34,0 \%)$ son más activas físicamente que las que nunca se han casado $(46,3 \%)$ y que las viudas o divorciadas. Las nunca casadas, por otra parte, tienen mayores probabilidades de permanecer sentadas durante sus tareas cotidianas habituales.

En lo que respecta al lugar de residencia, los adultos que viven en el Sur de Estados Unidos se muestran menos propensos a ser físicamente activos que los que residen en las demás zonas del país.

\section{Limitaciones del informe}

El informe del USDHHS posee algunas limitaciones. Una de ellas radica en la redacción de algunas de las preguntas relacionadas con las activi- dades en horas de recreo, que podría haber llevado a algunas personas a proporcionar en ese rubro datos acerca de su actividad física en otros momentos. Otra carencia es que las preguntas obligan al encuestado a calcular con rapidez en la mente la frecuencia y duración promedio de sus distintas actividades físicas, así como su intensidad, lo cual podría haber dado lugar a imprecisiones en la información aportada. Asimismo, las preguntas acerca de las tareas cotidianas habituales son de carácter general y cualitativo, de tal forma que el tiempo dedicado a caminar, permanecer sentado o de pie, y cargar o levantar bultos se basa en un cálculo subjetivo e impreciso, al igual que el peso de los bultos cargados durante dichas tareas.

\section{Utilidad del informe}

Pese a sus limitaciones, la NHIS aporta datos muy reveladores que serán empleados para observar el ritmo de los adelantos hacia la consecución de los objetivos de salud nacionales en materia de actividad física en adultos establecidos para el año 2010. Además, gracias al desglosamiento de los datos según características demográficas y socioeconómicas, el informe del USDHHS permite identificar a aquellos grupos de la población en los que deben concentrarse las actividades educativas y de promoción de la salud encaminadas a lograr un incremento de la actividad física en adultos.

El informe aquí descrito se compone de 24 varias páginas de texto corrido y de 10 páginas de cuadros donde los datos se presentan desglosados por numerosas categorías socioeconómicas y demográficas, como se explicó anteriormente. El informe completo puede verse en formato PDF o bajarse gratuitamente del sitio web del Centro Nacional de Estadísticas Sanitarias [National Center for Health Statistics] de los CDC. La dirección en Internet es http://www.cdc.gov/nchs/data/ad/ad333.pdf

\section{SYNOPSIS}

\section{Report looks at physical activity in both work and leisure time in the United States}

A recent report that bears the title Physical Activity Among Adults: United States, 2000 describes one of the few studies in the world that quantify total daily energy expenditure among adults in a given population. Most studies that attempt to assess the level of physical activity performed by a country's inhabitants look at leisure-time physical activity or energy expenditures while on the job. By looking at just one of those two areas, such studies provide only a partial view of the overall physical activity levels within a popula- 
tion. The report on the adult population of the United States of America, which is based on the National Center for Health Statistic's National Health Interview Survey for the year 2000, draws on data obtained through approximately 39000 household interviews, covering a total of more than 100000 individuals from all 50 states.

In essence, indicators were developed, based on frequency and duration, for regular leisure-time physical activity (exercise, sports, or active hobbies) as well as for the amount of "usual daily activity" (i.e., commuting, performing household chores, running errands, and engaging in other activities not performed during leisure time). Regular leisuretime physical activity was thus defined as light or moderate activity for a minimum of 30 minutes per session at least five times a week, or intense activity for a minimum of 20 minutes per session at least three times a week. Light or moderate activity was defined as activity lasting at least $10 \mathrm{mi}$ nutes that caused light sweating and a slight to moderate increase in breathing or heart rate. Vigorous activity was defined as activity lasting at least 10 minutes that caused heavy sweating or large increases in breathing or heart rate. In the case of "usual daily activity," its magnitude was determined by the number of times a day an individual had to walk or to lift or carry light, moderate, or heavy loads. These two indicators-regular leisure-time physical activity and usual daily activity - were combined to obtain an indicator for "overall physical activity level."

According to results, $19 \%$ of U.S. adults engage in a high level of physical activity, with men being more likely than women to engage in such activity. In both sexes, however, the level of physical activity declines with age, so that adults in the oldest age group (65 or older) were about five times as likely as adults in the youngest age group (18-24 years) to never be physically active. Women were also more likely than men to never engage in physical activity.

One in every 4 adults in the United States engages in little physical activity or none. Those who are more active in their usual daily activities are also more likely to engage in regular physical activity during leisure time. Almost three fourths of adults lift or carry light to heavy loads during their usual activities.

The report also examines the association between various socioeconomic and demographic variables and physical activity. Adults with incomes below the poverty level are three times more likely to be physically inactive than adults in the highest income group (4 times the poverty level or more). Around $15 \%$ of Hispanics, $14 \%$ of blacks, and $20 \%$ of whites engage in a high level of physical activity. In terms of education, individuals having an advanced degree are almost twice as likely as individuals without a high school diploma to have a high level of physical activity.

In short, the report described herein provides an accurate assessment of overall physical activity among U.S. adults and identifies high-risk population subgroups that should be the target of health promotion activities geared toward increasing physical activity levels. The data it includes will be the basis for monitoring progress toward achieving the 2010 national health objectives for physical activity among adults in the United States.

\section{REFERENCIAS}

1. Monteiro CA, Conde WL, Matsudo SM, Matsudo VR, Bonseñor IM, Lotufo PA. A descriptive epidemiology of leisure-time physical activity in Brazil, 1996-1997. Rev Panam Salud Publica 2003;14(4): 246254.
2. Hernández B, de Haene J, Barquera $S$, Monterrubio E, Rivera J, Shamah T, et al. Factores asociados con la actividad física en mujeres mexicanas en edad reproductiva. Rev Panam Salud Pública 2003 14(4):235-245.
3. Gentleman JF, Pleis JR. The National Health Interview Survey: an overview. [Sitio de Internet del American College of Physicians]. Se encuentra en: http:// www.acponline.org/journals/ecp/ mayjun02/ gentleman.htm . Acceso obtenido el 21 de octubre de 2003. 